\title{
Is there a PSA cut-off value indicating incidental prostate cancer in patients undergoing surgery for benign prostatic hyperplasia?
}

\author{
Senol Tonyali ${ }^{1}$, Cavit Ceylan ${ }^{2}$, Erdogan Aglamis ${ }^{3}$, Serkan Dogan ${ }^{4}$, Sedat Tastemur ${ }^{2}$, \\ Mustafa Karaaslan ${ }^{2}$ \\ ${ }^{1}$ Department of Urology, Istanbul University Istanbul School of Medicine, Istanbul, Turkey; \\ 2 Department of Urology, University of Health Sciences, Ankara City Hospital, Ankara, Turkey; \\ ${ }^{3}$ Department of Urology, University of Health Sciences, Elazig City Hospital, Elazig, Turkey; \\ ${ }^{4}$ Department of Urology, Sancaktepe Sehit Prof. Dr. Ilhan Varank Training and Research Hospital, Istanbul, Turkey.
}

\begin{abstract}
Summary Aim: To investigate incidental prostate cancer (IPCa) rate and to determine prostate specific antigen (PSA) cut-off value indicating PCa in patients who underwent surgery by being diagnosed with benign prostatic hyperplasia (BPH) clinically or by standard prostate biopsy. Methods: Data of 317 patients, who underwent transurethral resection of the prostate (TURP) or open prostatectomy (OP) with pre-diagnosis of $B P H$, were evaluated retrospectively. The examined parameters included patients' demographics, preoperative serum PSA values, digital rectal examination (DRE) findings, surgical method, histopathological findings and Gleason Scores.

Results: A total of 317 patients were included the study.
\end{abstract} The median age of patients was 69 years (min: 51-max: 79) and the median PSA value was $3.24 \mathrm{ng} / \mathrm{dl}$ (min: 0.17-max: 34.9). In 21 patients (6.6\%); DRE findings were in favor of malignancy, but prostate biopsy resulted as BPH. While 281 (88.6\%) of the patients underwent TURP, 36 (11.4\%) underwent open prostatectomy. PCa was detected in 21 (6.6\%) patients.

PSA was statistically higher in patients who underwent OP compared to patient who underwent TUR-P, 5.9 (min: 1.2 max: 27.6, IR: 8.7) vs. 2.8 (min: 0.1-max: 34.9, IR: 4.2) ng/dl, $p<.001$. The rate of IPCa among four PSA group was similar $(p=0.46)$. There was no difference between the rate of IPCa in patients younger and older than 70 years, $(p=0.11)$. Please change whole sentence as 'The median PSA level was slightly higher in patients diagnosed with BPH compared to patients diagnosed with IPCa, 3.2 (min: 0.1-max: 34.9) vs. 2.7 (min: 0.3-max: 26.5) $n g / d L, p=0.9$.

Conclusions: IPCa still remains an important clinical problem. We were not able to find any correlation of PSA and age with incidental PCa.

KEY WORDS: Incidental; Prostate cancer; Open prostatectomy.

Submitted 19 October 2020; Accepted 1 December 2020

\section{INTRODUCTION}

Prostate cancer ( $\mathrm{PCa}$ ) is the most commonly diagnosed cancer in men and the most common cause of cancer deaths following lung cancer. According to autopsy studies, the risk of PCa in men $>50$-year-old is about $30 \%(1,2)$.
Surgical treatment remains the most effective option in patients with lower urinary tract obstructive symptoms who did not respond to medical therapy and have moderate to severe symptoms. Evaluation of prostate specific antigen (PSA) and digital rectal examination (DRE) constitute the routine urological evaluations before surgery for benign prostatic hyperplasia (BPH) to exclude PCa; PSA density, PSA velocity, free/total PSA and PSA according to age could be utilized if required. A transrectal ultrasound-guided prostate biopsy is the gold standard to rule out PCa in these patients and have been used extensively. Neoplastic tissue may not be detected in sample when biopsy is performed with transrectal ultrasound (TRUS) guidance, even if the PSA levels are high or DRE findings are positive (11).

Incidental prostate cancer (IPCa) is the diagnosis of PCa with histopathological examination of resected prostate tissue, which was previously considered benign. IPCa rates after both open prostatectomy (OP) and transurethral resection of prostate (TURP) have been reported to be vary between 5\% and $41 \%$ in the literature (3-8). Despite normal PSA values, DRE findings, and normal prostate biopsy results before surgery; IPCa is still a challenge for physicians and patients with high expectations. In this study, we aimed to evaluate rate of IPCa after benign prostate surgery and to determine if there is a PSA cut-off value indicating IPCa in Turkish population.

\section{MATERIALS AND METHODS}

The study protocol was approved by the Institutional Review Board of Turkiye Yuksek Ihtisas Training and Research Hospital (Approval Number: 15.03.201829620911-929-E.2475).

The data of all consequent patients who underwent TURP or OP with the pre-diagnosis of BPH between 2008-2018 were evaluated retrospectively. Patients with normal DRE and PSA levels in preoperative evaluation as well as patients who underwent TRUS guided prostate needle biopsy prior to surgery due to abnormal DRE and/or high serum PSA values and were reported to have not PCa were included the study. Patients who had a history of $\mathrm{PCa}$ or patients who received any treatment

No conflict of interest declared. 
due to PCa were excluded from the study. The examined parameters included patients' demographics, preoperative serum PSA values, DRE findings, surgical method, histopathological findings and Gleason Scores.

The relationship between these parameters and IPCa was investigated. Tha patients were divided into 4 groups according to PSA values: Group 1: PSA $<2.5 \mathrm{ng} / \mathrm{dL}$, Group 2: PSA = 2.5-4 ng/dL, Group 3: PSA $=4.10 \mathrm{ng} / \mathrm{dL}$, Group 4: PSA > 10 ng/dL.

\section{Statistical analysis}

Statistical analysis was performed using IBM SPSS statistical package program v 21.0. Continues variables were given in median (minimum-maximum) and categorical variables were given in numbers and percentage. ROC curve analysis was used to determine cut-off value for PSA. Spearman correlation test was performed for the relationship between PSA, age and IPCa. Chi-Square test was used to determine the differences between frequencies in two categorical variables. Mann-Whitney $U$ test was used to compare the age and PSA of the patients with and without prostate cancer when there was not a normal distribution after Kolmogorov-Smirnov test.

\section{Results}

A total of 317 patients were included the study.

The median age of patients was 69 years (min: 51, max: 79 ) and the median PSA value was $3.24 \mathrm{ng} / \mathrm{dl}$ (min: 0.17, max: 34.9). In 21 patients (6.6\%) DRE findings were in favor of malignancy, but prostate biopsy resulted as BPH. While 281 (88.6\%) of the patients underwent TURP, 36 (11.4\%) underwent open prostatectomy. PCa was detected in $21(6.6 \%)$ patients (Table 1).

The Gleason scores of the patients with IPCa were as follow: G6 $(n=10), G 7(3+4)(n=2), G 7(4+3)(n=1)$, G8 $(n=2)$, G9 $(n=3)$ and G10 $(n=3)$. Four patients with Gleason scores $>6$ underwent radical prostatectomy. A patient in high-risk group after this radical prostatectomy and a patient with low-surgical performance

\section{Table 1.}

Patient characteristics and comparison of patients with and without incidental prostate cancer.

\begin{tabular}{|c|c|c|c|c|c|}
\hline & & $\begin{array}{c}\text { Total } \\
(n=317)\end{array}$ & $\begin{array}{c}\text { Benign prostate } \\
\text { hyperplasia }(n=296)\end{array}$ & $\begin{array}{c}\text { Incidental prostate } \\
\text { cancer }(\mathrm{n}=21)\end{array}$ & $P$ value \\
\hline Median age (years) & $\begin{array}{l}\text { Age }<70 \text { years } \\
\text { Age }>70 \text { years }\end{array}$ & $\begin{array}{c}69(51-79) \\
178 \\
139\end{array}$ & $\begin{array}{l}170 \\
126\end{array}$ & $\begin{array}{c}8 \\
13\end{array}$ & $0.11^{\wedge}$ \\
\hline Median PSA (ng/dL) & $\begin{array}{c}\text { PSA }<2.5 \\
\text { PSA: } 2.5-4.0 \\
\text { PSA: } 4-10 \\
\text { PSA }>10\end{array}$ & $\begin{array}{c}3.2(0.1-34.9) \\
118(37.2 \%) \\
73(23 \%) \\
93(29.3 \%) \\
33(10.4 \%)\end{array}$ & $\begin{array}{l}109 \\
71 \\
85 \\
31\end{array}$ & $\begin{array}{l}9 \\
2 \\
8 \\
2\end{array}$ & $0.46^{\wedge}$ \\
\hline Gleason score & $\begin{array}{l}\text { Gleason } 3+3 \\
\text { Gleason } 3+4 \\
\text { Gleason 4+3 } \\
\text { Gleason 4+4 } \\
\text { Gleason 4+5 } \\
\text { Gleason 5+4 } \\
\text { Gleason 5+5 } \\
\text { Gleason 5+3 }\end{array}$ & $\begin{array}{l}10(3.15 \%) \\
2(0.63 \%) \\
1(0.31 \%) \\
1(0.31 \%) \\
2(0.63 \%) \\
1(0.31 \%) \\
3(0.94 \%) \\
1(0.31 \%)\end{array}$ & & & \\
\hline
\end{tabular}

who had a Gleason score $>9$ were referred to the oncology clinic for adjuvant hormone therapy and radiotherapy. PSA was statistically higher in patients who underwent OP compared to patient who underwent TUR-P, 5.9 (min: 1.2- max: 27.6, IR: 8.7) vs. 2.8 (min: 0.1-max: 34.9, IR: 4.2) $\mathrm{ng} / \mathrm{dl}, \mathrm{p}<.001$. The rate of IPCa among four PSA group was similar $(\mathrm{p}=0.46)$. There was no difference between the rate of IPCa in patients younger and older than 70 years, $(p=0.11)$. The median PSA level was slightly higher in patients diagnosed with BPH compared to patients diagnosed with IPCa, 3.2 (min: 0.1-max: 34.9) vs. 2.7 (min: 0.3-max: 26.5) ng/dL, $\mathrm{p}=0.9$.

\section{Discussion}

Today, the rate of IPCa is still found to be high in patients diagnosed with BPH clinically or after prostate biopsy under the guidance of TRUS. The widespread use of new biopsy techniques such as multiparametric magnetic resonance imaging (MRI) - targeted biopsy may decrease the rate of IPCa. In our study, 6.6\% IPCa was detected in patients who underwent open prostatectomy or TUR-P with the diagnosis of benign prostatic hyperplasia.

Prostate cancer incidence and cancer-related mortality are more common in European countries than in Asian countries $(12,13)$. The rate of diagnosis of IPCa reaches up to $16 \%$ in parallel with the increase in life expectancy and might not to be underestimated (5). The use of PSA and its derivatives in daily urology practice, the increase in the number of prostate needle biopsy cores and the improvement in technical methods have decreased the rate of IPCa cases over the years but it has not been minimalized (5). Rohr et al. reported a rate of 15\% IPCa in 457 TURP operations in 1987 and Merril et al., reported in their study that the rate of IPCa determined by TURP gradually decreased between 1980 and 1999. They also reported that IPCa rates decreased from $39 \%$ to $7 \%$ from 1980 s to $2000(14,15)$. In a study that emphasized the importance of age-specific PSA, IPCa was observed in $13 \%$ of patients who underwent TURP and open prostatectomy, whereas this rate was decreased to $6.3 \%$ with age-specific PSA assessment (16). In a study by Zigeuner et al., it was reported that preoperative DRE and PSA test combination caused a 50\% reduction in the detection rate of IPCa (16). In our study, we detected IPCa in $6.6 \%$ of patients that underwent TURP and open prostatectomy. Our rate is consistent with the literature and we think that the decrease in the rate of IPCa is related to early diagnosis with the advances in diagnostic tests and biopsy techniques we utilized in the light of the technological developments. On the other hand, with increase in life expectancy, it is obvious that IPCa that can be newly diagnosed is that was previously named as hidden prostate cancer. Perhaps the rate of $19.9 \%$ IPCa detected by Abedi et al. might be the best example of this. This demonstrates that IPCa diagnosis rate cannot be minimized and is not constantly reduced (4). The lack of 
homogeneous study cohorts and inter-racial differences might explain the wide range of this rate in literature.

Zigeuner et al., reported an IPCa of $7.9 \%$ in 445 patients who underwent TURP or open prostatectomy after transrectal prostate biopsy due to high PSA level and/or abnormal DRE. The rate of IPCa reported in this study is similar to our results, however the inclusion of patients who underwent transrectal prostate biopsy prior to surgery might affect obtaining a low IPCa rate (17).

In a study conducted by Otto et al., 771 patients who underwent TURP were retrospectively analyzed and IPCa was detected in 11 patients (1.4\%). Among 11 patients, ten had a (91\%) Gleason Score of $3+3=6$ and one (9\%) patient had a Gleason score of $3+4=7$. Of 11 patients with IPCa, nine had Tla disease and two had Tlb disease. (18). When compared with this study, the rate of IPCa was found to be higher in our study. However, in their study, Otto et al., only included patients who underwent TURP and the mean resected tissue weight was $8.1 \mathrm{~g}$.

The amount of prostate sample examined may affect the ratio of IPCa. In our study, $47.6 \%$ of IPCa cases had a Gleason Score of $\leq 6$, which is lower than the rate reported by Otto et al.

In another study by Abedi et al., authors reported higher IPCa rates detected via open prostatectomy compared to TURP (4). Although, IPCa ratios detected via open prostatectomy were higher compared to TURP in our study, it was not statistically significant. This might be due to the low number of patients in the open prostatectomy group. In PCa diagnosis, various methods have been developed to prevent unnecessary repeated biopsies and to detect $\mathrm{PCa}$ that need to be treated. Multiparametric magnetic resonance imaging (MRI) fusion biopsy is one of these methods gaining importance day by day (19). Several researchers reported that MRI-targeted biopsy is superior to standard transrectal ultrasonography (TRUS)-guided biopsy in detection of PCa $(20,21)$. European Association of Urology (EAU) guidelines favor MRI guided biopsy to systematic biopsy in detecting ISUP grade $\geq 2 \mathrm{PCa}$ in the repeated-biopsy setting. However, in biopsy-naive patients this difference was stated to be less significant (22). In their retrospective multicenter study, Porreca et al. investigated the utility of 'in-bore' MRI prostate biopsy to exclude significant PCa in patients with BPH scheduled for transurethral laser enucleation of prostate.

The authors concluded that including mpMRI and MRI guided biopsy prior to surgery for BPH might lead to low PCa and avoid unnecessary standard TRUS-guided biopsies (23). In our study, prostate biopsy was performed only with conventional TRUS which might be considered a limitation of our study.

Incidental PCa detection still remains as a troublesome problem for the patients, urologists and the pathologists. According to the Collage of American Pathologists statement, it was suggested to sample all prostate tissue left behind when Tla PCa was detected (8). In routine practice of pathologists, it is not possible to sample all of the TURP or open prostatectomy materials. This may cause IPCa cases to be overlooked. For this reason, the determination of preoperative clinical parameters that may predict IPCa may change the preoperative approach of the urologists, and may also help the pathologists to determine the required pathological sample amount for an accurate examination. However, this may also lead to over diagnosis and over treatment risk. Our study is not without limitations. The retrospective nature of our study and the inclusion of patients treated with different surgical methods are the main limitations of our study. In addition, the absence of patients to whom multiparametric MRI-targeted biopsy was applied might be another issue.

Prospective multicenter studies with large patient number comparing standard TRUS prostate biopsy and new biopsy techniques such as MRI-targeted prostate biopsy might improve our knowledge in this topic.

\section{Conclusions}

Incidental PCa detection rate is considerably high in patients undergoing surgery for benign prostatic hyperplasia, which was diagnosed clinically or via standard TRUS-guided prostate needle biopsy. Thus, IPCa still remains an important clinical problem. We were not able to find any correlation of PSA and age with incidental PCa. Determination of new parameters that can predict IPCa detection and the widespread use of new biopsy techniques such as MRI-targeted biopsy may decrease the rate of IPCa.

\section{REFERENCES}

1. Jemal A, Murray T, Ward E, et al. Cancer statistics. CA Cancer J Clin. 2005; 55:10-30.

2. Stangelberger A, Waldert M, Djavan B. Prostate cancer in elderly men. Rev Urol. 2008; 10:111-119.

3. Yoo C, Oh CY, Kim SJ, et al.. Preoperative clinical factors for diagnosis of incidental prostate cancer in the era of tissue-ablative surgery for benign prostatic hyperplasia: a Korean multi-center review. Korean J Urol. 2012; 53:391-395.

4. Abedi AR, Fallah-Karkan M, Allameh F, et al. Incidental prostate cancer: a 10-year review of a tertiary center, Tehran, Iran. Res Rep Urol. 2018; 10:1-6.

5. Stamey TA, Kabalin JN, McNeal JE. Prostate specific antigen in the diagnosis and treatment of adenocarcinoma of prostate. J Urol. 1989; 141:1076.

6. Voigt S, Hüttig F, Koch R, et al. Risk factors for incidental prostate cancer who should not undergo vaporization of the prostate for benign prostate hyperplasia? Prostate. 2011; 71:1325-1331.

7. Helfand BT, Anderson CB, Fought A, et al. Postoperative PSA and PSA velocity identify presence of prostate cancer after various surgical interventions for benign prostatic hyperplasia. Urology. 2009; 74:177-183.

8. Trpkov K, Thompson J, Kulaga A, Yilmaz A. How much tissue sampling is required when unsuspected minimal prostate carcinoma is identified on transurethral resection? Arch Pathol Lab Med. 2008; 132:1313-6.

9. Jahn JL, Giovannucci EL, Stampfer MJ. The high prevalence of undiagnosed prostate cancer at autopsy: implications for epidemiology and treatment of prostate cancer in the prostate-specific antigen-era. Int J Cancer. 2015; 137:2795-2802.

10. McConnell JD. Epidemiology, etiology, pathophysiology, and diag- 
nosis of benign prostatic hyperplasia. In: Walsh PC, Retik $A B$, Vaughan Jr ED, Wein AJ, eds. Campbell's Urology. 7thed. Philadelphia: WB Saunders Company. 1998, p.1429-52.

11. Fernández RE, Gómez VF, Alvarez CL, et al. Clinicopathological study of incidental cancer prostate in patients undergoing surgery for symptomatic diagnosis of BPH. Actas Urol Esp. 2006; 30:33-37

12. Parkin DM, Bray F, Ferlay J, Pisani P. 2005. Global cancer statistics. CA Cancer J Clin. 2002; 55:74-108.

13. Landis SH, Murray T, Bolden S, Wingo PA. Cancer statistics. CA Cancer J Clin. 1999; 49:8-31.

14. Rohr LR. Incidental adenocarcinoma in transurethral resections of prostate. Am J Surg Pathol. 1987; 11:53-8.

15. Merril RM, Wiggins CL. Incidental detection of population based prostate cancer incidence rates trough transurethral resection of the prostate. Urol Oncol. 2002; 7:213-9.

16. Zigeuner RE, Lipsky K, Rieder I, et al. Did the rate of incidental prostate cancer change in the era of PSA testing? A retrospective study of 1127 patients. Urology; 2003; 62:451-5.

17. Zigeuner R, Schips L, Lipsky K, et al. Detection of prostate cancer by TURP or open surgery in patients with previously negative transrectal prostate biopsies. Urology; 2003; 62:883-887.
18. Otto B, Barbieri C, Lee $R$, et al. Incidental prostate cancer in transurethral resection of the prostate specimens in the modern era. Adv Urol. 2014; 2014:627290.

19. Borkowetz A, Platzek I, Toma M, et al. Comparison of systematic transrectal biopsy to transperineal MRI/ultrasound-fusion biopsy for the diagnosis of prostate cancer. BJU Int. 2015; 116: 873-9.

20. Kasivisvanathan V, Rannikko AS, Borghi M et al. MRI-targeted or standard biopsy for prostate-cancer diagnosis. N Engl J Med, 2018; 378:1767-77.

21. Kasivisvanathan V, Stabile A, Neves JB et al. Magnetic resonance imaging-targeted biopsy versus systematic biopsy in the detection of prostate cancer: a systematic review and meta-analysis. Eur Urol. 2019; 76:284-303.

22. Mottet N, Cornford P, van der Bergh RCN, et al. EAU- EANMESTRO- ESUR- SIOG Guidelines of Prostate Cancer 2020. Available at: https://uroweb.org/guideline/prostate-cancer/

23. Porreca A, D'Agostino D, Vigo M, et al. "In-bore" MRI prostate biopsy is a safe preoperative clinical tool to exclude significant prostate cancer in symptomatic patients with benign prostatic obstruction before transurethral laser enucleation. Arch Ital Urol Androl. 2020; 91:224-229.

\section{Correspondence}

Senol Tonyali, MD (Corresponding Author)

senoltonyali@hotmail.com

Cavit Ceylan, MD

ceylancavit@yahoo.com

Sedat Tastemur, MD

sedattastemur@yahoo.com

Department of Urology, Istanbul University Istanbul School of Medicine Surgery Monobloc Floor:1, 34104 Çapa Fatih, Istanbul (Turkey)

Mustafa Karaaslan, MD

mustafakaraaslan23@gmail.com

Erdogan Aglamis, MD

uroloji23@yahoo.com

Department of Urology, University of Health Sciences, Elazig City Hospital, Elazig (Turkey)

\section{Serkan Dogan, MD}

sdogan1907@yahoo.co.uk

Department of Urology, Sancaktepe Sehit Prof. Dr. Ilhan Varank Training and Research Hospital, Istanbul (Turkey) 